\title{
IMPORTANCE OF CULTURE AND SENSITIVITY FOR BETTER HEALING OF A MASTOID CAVITY
}

\author{
Shweta Baviskar ${ }^{1}$, Laveena Mehta², Tushar Gori ${ }^{3}$,Vrushali Vishal Kulkarni', Milind Ubale ${ }^{5}$, Poorva Sule
}

${ }_{1}^{1}$ Assistant Professor, Department of ENT, RGMC, Kalwa, Mumbai, Maharashtra, India.

${ }_{2}^{2}$ Registrar, Department of ENT, RGMC, Kalwa, Mumbai, Maharashtra, India.

3 Postgraduate Student, Department of ENT, RGMC, Kalwa, Mumbai, Maharashtra, India.

${ }^{4}$ Assistant Professor, Department of Community Medicine, RGMC, Kalwa, Mumbai, Maharashtra, India.

${ }^{5}$ Professor and HOD, Department of Microbiology, RGMC, Kalwa, Mumbai, Maharashtra, India.

${ }^{6}$ Assistant Professor, Department of Microbiology, RGMC, Kalwa, Mumbai, Maharashtra, India.

\section{BACKGROUND}

ABSTRACT

Determination of the type of microorganisms, which grow in the middle ear can help determine the appropriate antibiotic to be administered orally as well as topically. In an era of growing antibiotic sensitivity, it becomes prudent to be judicious with prescribing antibiotics. This can be easily done with the help of an ear swab. This has a positive effect in faster healing of wide mastoid cavity and can prevent the problems of a discharging cavity post-operatively.

\section{MATERIALS AND METHODS}

A randomised controlled trial study was conducted in the Department of ENT at Rajiv Gandhi Medical College, Kalwa from July 2017 to December 2017 on 50 patients. The patients were selected by simple random sampling method. An ear swab is first taken prior to posting the patient for a tympanomastoid surgery and following the surgery a swab is retaken on the 7th post-operative day and antibiotics may be changed based on the reports obtained.

\section{RESULTS}

During post-operative 3rd week visit of all 50 patients, it was observed that out of the 25 patients treated on the basis of pus culture and sensitivity reports. 23 patients (92\%) responded well with clean mastoid cavity and improved healing to the point of epithelialisation, whereas out of the remaining 25 patients treated empirically only 9 patients (36\%) responded well with clean mastoid cavity and improved healing and the rest followed up with either discharging cavities or granulation tissue.

\section{CONCLUSION}

Use of ear swab and its role in selection of antibiotics has a positive effect in faster healing of wide mastoid cavity and can prevent the problems of a discharging cavity post-operatively.

\section{KEY WORDS}

Cholesteatoma, Antibiotic, Resistance, Unsafe CSOM.

HOW TO CITE THIS ARTICLE: Baviskar S, Mehta L, Gori T, et al. Importance of culture and sensitivity for better healing of a mastoid cavity. J. Evolution Med. Dent. Sci. 2018;7(30):3390-3393, DOI: 10.14260/jemds/2018/765

\section{BACKGROUND}

CSOM with cholesteatoma, widely known as the unsafe type of CSOM, is a common presentation to an ENT surgeon. Patients with this disease usually present with discharging ear, which is often persistent post-operatively. It is therefore necessary to try and standardise the management of not only their disease but also of the clinical presentations. In today's era of continuously evolving microorganisms, it is common to see decreased response to standard drugs of choice in patients of CSOM. While CSOM can be attributed to multiple factors, role of microorganisms and their response to various drugs has gained a lot of popularity. The presence of biofilms, which cause resistance to antibiotics, is a major contributor in persistence of disease.(1) It is thus important to have a thorough understanding of the species isolated in cases of

'Financial or Other Competing Interest': None.

Submission 09-06-2018, Peer Review 11-07-2018,

Acceptance 17-07-2018, Published 23-07-2018.

Corresponding Author:

Dr. Shweta Baviskar,

\#1602, Tower 4, Regency Heights,

Brahmand, Thane-West,

Mumbai, Maharashtra, India.

E-mail: shweta.katna@gmail.com

DOI: $10.14260 /$ jemds $/ 2018 / 765$
CSOM and their response to various antibiotics.(2) In patients who have undergone mastoidectomy surgeries, continued discharge is not only attributed to large mastoid cavity but also to the constantly changing microorganisms and their developing resistance to antibiotics routinely prescribed by the ENT surgeon.

\section{MATERIALS AND METHODS}

A randomised controlled trial study was conducted in the Department of ENT at Rajiv Gandhi Medical College, Kalwa from July 2017 to December 2017 on 50 patients. The sample size has been limited for our convenience. The first 50 patients with unsafe CSOM, who gave consent for further management were included in the study. All patients who presented to the ENT OPD with unsafe disease were informed about the nature of their disease and the management required including the surgery and possible complications. Each patient was first examined with Otoscope with 2 Diopter magnification and the disease was established. A sterile ear swab of 25 randomly selected patients was taken and sent for microbiological examination. The patients were selected by simple random sampling method. All the patients were then on oral and topical antibiotics. The remaining 25 patients were empirically started on T. Augmentin. A HRCT temporal bone was obtained, and the patient was posted for 
surgery the next day. A tympanomastoid surgery was performed for each patient and the cavity was cleared of any disease. The patients were discharged on $2^{\text {nd }}$ post-operative day and asked to follow-up on post-op day 8 for suture removal. The same 25 out of 50 patients whose pre-operative swabs were taken were also asked to follow-up on post-op day 10 for a repeat swab for culture and sensitivity testing. Antifungal ear drops were administered to all patients. The culture samples of 25 patients were inoculated on routine bacterial culture media. Sabouraud's medium was used for mycotic pathogens in aerobic incubation. Robertson's cooked medium was used for anaerobic bacteria and was incubated in McIntosh Filde's jar.

\section{Inclusion Criteria}

Patients with unsafe CSOM of age group: 5 - 40 years with patients of bilateral ear disease with complications of CSOM.

\section{Exclusion Criteria}

Cases of pregnant women.

The culture reports were awaited for 3 days and antibiotics were prescribed based on the culture and sensitivity report of the post-op cavity for 25 patients. For the remaining 25 patients, empirical antibiotic therapy was started. A regular cleaning of the cavity was done for all patients on each visit. Cavity was observed for signs of epithelialisation.

\section{Statistical Analysis}

Proportions of clean mastoid cavity were calculated, and analysis was carried out with the statistical software SPSS 20. Significant difference in proportions was checked.

Statistical Z-test for (SEDP) standard error of difference of proportions was applied with $5 \%$ level of significance.

\section{RESULTS}

A group of 50 patients with unsafe CSOM were included in the study. Both males $(60 \%)$ and $40 \%$ females were part of the study with a slight predominance in males.

\section{Genderwise distribution}

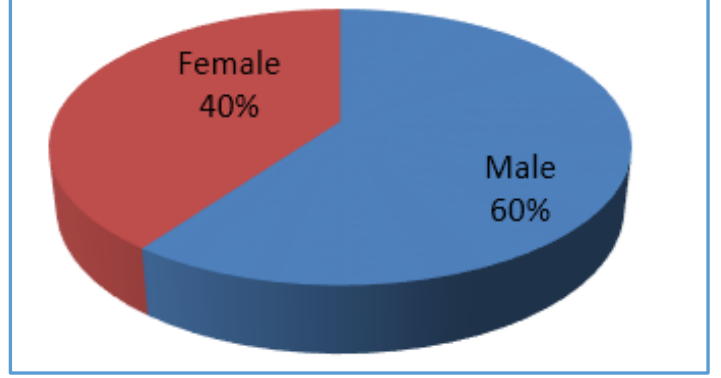

Patients were within the age group of 5 to 40 years with most patients presenting in the $3^{\text {rd }}$ decade of life.

\begin{tabular}{|c|c|c|}
\hline Age Group & Number & Percentage \\
\hline $5-10$ & 8 & $16 \%$ \\
\hline $11-20$ & 7 & $14 \%$ \\
\hline $21-30$ & 14 & $28 \%$ \\
\hline $31-40$ & 21 & $42 \%$ \\
\hline \multicolumn{3}{|c|}{ Table 1 } \\
\hline
\end{tabular}

A culture swab was taken prior to surgery and again on the $10^{\text {th }}$ post-operative day for 25 randomly selected patients. Among the strains isolated, the most common was Pseudomonas aeruginosa (48\%) followed by Staphylococcus aureus closely followed by Methicillin-Resistant Staphylococcal aureus.

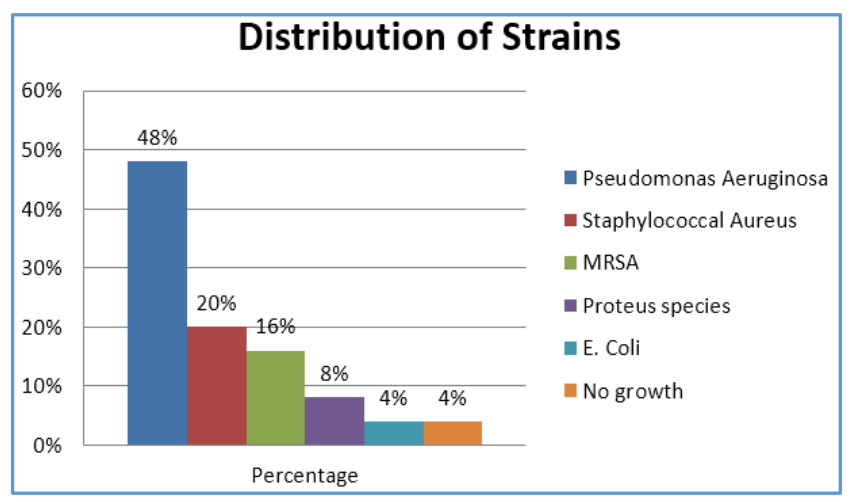

Antibiotic sensitivity was tested for and results were assessed.

\begin{tabular}{|c|c|c|c|c|c|c|c|}
\hline Organism & Pen & Cot & Aug & Cholph & Cip & Genta & Cefta \\
\hline $\begin{array}{c}\text { Pseudomonas } \\
\text { aeruginosa }\end{array}$ & - & - & - & $72.1 \%$ & $95.6 \%$ & $88.3 \%$ & $100 \%$ \\
\hline $\begin{array}{c}\text { Staphylococcal } \\
\text { aureus }\end{array}$ & $60 \%$ & $68.6 \%$ & $75.3 \%$ & $86 \%$ & $92 \%$ & $90 \%$ & - \\
\hline MRSA & $100 \%$ & $73 \%$ & $97 \%$ & $66.3 \%$ & $85 \%$ & $87.9 \%$ & - \\
\hline $\begin{array}{c}\text { Proteus } \\
\text { species }\end{array}$ & - & $64.8 \%$ & $87.5 \%$ & $75.7 \%$ & $96 \%$ & $89.9 \%$ & $62 \%$ \\
\hline E. coli & - & $75 \%$ & $74.3 \%$ & $70 \%$ & $99.1 \%$ & $100 \%$ & $75 \%$ \\
\hline \multicolumn{7}{|c|}{ Table 2 } \\
\hline
\end{tabular}

Pen $=$ Penicillin, Cot $=$ Cotrimoxazole, Aug $=$ Augmentin, Cholph $=$ Chloramphenicol, $\mathrm{Cip}=$ Ciprofloxacin, Genta $=$ Gentamycin, Cefta= Ceftazidime.

In our study one group of 25 patients who have the pathogen studied had a dry cavity 3 weeks post-operatively and another 25 patients in other group were blindly started on empirical therapy had dry cavity 3 weeks post-operatively. Hence, two groups were tested with different mechanism.

During post-operative $3^{\text {rd }}$ week visit of all 50 patients, it was observed that out of the 25 patients treated on the basis of pus culture and sensitivity reports, 23 patients (92\%) responded well with clean mastoid cavity and improved healing to the point of epithelialisation, whereas out of the remaining 25 patients were treated empirically. Only 9 patients $(36 \%)$ responded well with clean mastoid cavity and improved healing and the rest followed up with either discharging cavities or granulation tissue.

To check the significant difference between proportions of patients with clean mastoid cavity in both methods, statistical test for (SEDP) standard error of difference of proportions was applied with $5 \%$ level of significance. The result is significant at LOS 0.05 (P-value $<0.00001$ ) implies that there is a significant difference between proportion of clean mastoid cavity in two methods namely pathogen study and empirical therapy.

\section{DISCUSSION}

The complaints of discharging ear in an operated mastoid cavity is an age-old problem. Current concepts favour the inside out mastoidectomy procedure as compared to the 
conventional canal wall down mastoidectomy. $(3,4)$ The idea of the inside-out technique is to limit the size of the cavity when possible and prevent the doctor dependence associated with large cavities.(5) Various studies have been conducted on the probable causes of discharge seen in post-operative cavities. Some have reported that the problems are merely due to the large cavity itself. This is because the larger the cavity, longer the time required for its epithelialisation.(6) Another cause for concern was the incomplete removal of the disease. $(7,8)$ However, the most important factor still remains to be presence of bacterial infection. ${ }^{(9)}$

This warrants a thorough study of the various microorganisms, which are cultivated from patients with CSOM including their sensitivity to drugs routinely administered. It is becoming increasingly common to see a change in the microorganisms, which are cultivated along. There is also a trend of increasing antibiotic sensitivity. This has led to suboptimal treatment of an operated mastoid cavity, due to which we routinely notice a "weeping" cavity.

However, this problem can be easily tackled with a simple, economical, non-invasive procedure of routinely taking an ear swab pre-operatively as well as postoperatively and administering antibiotics based on the sensitivity reports obtained. Multiple such studies have been conducted in different parts of the world, yielding similar results. The most common pathogen to be inoculated is Pseudomonas aeruginosa. $(6,10-12)$ The second most commonly inoculated pathogen was found to be Staphylococcus aureus. $(13,14)$

A noteworthy feature noticed by the authors of this study is an increase in the strains of MRSA. This evidence confirms the importance of culture and sensitivity report. While MRSA had a limited role in the contribution to CSOM in the past, its proliferation poses a serious threat to the healing of mastoid cavity.

Based on the P. aeruginosa routinely cultured, the drug of choice is quinolones.(15) Our study confirms that have this microorganism has a high sensitivity to Ciprofloxacin. However, these must be administered with caution in the paediatric age group, as there is confirmed evidence to suggest that they hamper growth. They have a limited role to play in children less than 13 years of age and should be used as judiciously as possible. The safest antibiotic in children still remains a combination of Amoxicillin and Clavulanic acid.(16)

For patients with Staph. aureus infection, quinolones still remain the most potent antibiotic.(17) Even patients with isolated strands of MRSA have shown to respond well to Ciprofloxacin, making it the drug of choice as an empirical treatment. (16)

Despite all the above evidence, the operating surgeon cannot afford to get complacent with the treatment of CSOM. There have been reports which have shown Quinolone resistance in patients with $P$. aeruginosa.(16) These may be attributed to a variety of factors, most importantly an abuse of antibiotics without evidence of the underlying microorganisms. (18)

Besides oral medications, topical antibiotics too have an important role to play in pre-operative and post-operative patients of unsafe CSOM.(16) An ideal ear drop should provide coverage for both gram positive as well as gram negative bacteria. A variety of aminoglycoside and quinolone ear drops can achieve this. Chloramphenicol is the most favoured aminoglycoside followed by Gentamicin. Antibiotic ear drops can be administered by itself or in combination with steroid ear drops. The disadvantage of aminoglycoside are potential vestibular and cochlear toxicity. This can be overcome by use of quinolones. Use of steroids like dexamethasone also aid in the treatment of granulation tissue and hypertrophied mucosa.(19)

The $\mathrm{pH}$ of ear drops with or without steroids are usually kept at an acidic $\mathrm{pH}$, which is the normal $\mathrm{pH}$ of the external auditory canal. The acidity of the ear drops may however lead to irritation and burning sensation once they reach the middle ear affecting the compliance of the patients, especially in children. Moreover, due to the high viscosity, there is a sedimentation of ear drops in the external auditory canal which can prevent the action of the antibiotic within. This is one of the identified causes of persistent ear discharge.

This can be combated with the use of acetic acid ear drops, which can penetrate through the tenacious discharge and drops and create a pathway for the action of antibiotics. Studies have been conducted which have shown that even in cases of resistant pseudomonas and staphylococcal infections, quinolone ear drops have proved to be efficacious.(20)

\section{CONCLUSION}

Unsafe CSOM is a common problem, which requires management in the form of a large tympanomastoid cavity. Ear discharge in large cavities may be due to persistent bacterial infections, despite administration of antibiotics. In an era of constantly evolving microorganisms, it becomes necessary to isolate the pathogen responsible for the disease. This in turn will help in proper selection of the antibiotic to be administered. From this study, we conclude that Ciprofloxacin is the drug of choice in adult patients presenting with unsafe CSOM, which can be administered via oral as well as topical route. The antibiotic can be continued for a period of 10 days post-operatively, after which the antibiotic should be changed based on the culture and sensitivity report. According to this study, this increasing evidence of MRSA and studies confirming resistance to quinolones confirms the need for culture swabs from ear, both prior to and after surgery. This might be an effective way to prevent further resistances of the microbes as well as help in subduing the discharge traditionally associated with large cavities.

$92 \%$ of patients who have the pathogen studied had a dry cavity 3 weeks post-operatively. $36 \%$ of patients who were blindly started on empirical therapy had dry cavity 3 weeks post-operatively. Study also proved that the role of preoperative culture and sensitivity swabs is showing significant result in pathogen study as compared to empirical therapy had dry cavity 3 weeks post-operatively.

\section{REFERENCES}

[1] Stewart PS, Costerton JW. Antibiotic resistance of bacteria in biofilms. Lancet 2001;358(9276):135-8.

[2] Hall-Stoodley L, Stoodley P. Evolving concepts in biofilm infections. Cell Microbiol 2009;11(7):1034-43.

[3] Chamoli P, Singh CV, Radia S, et al. Functional and anatomical results of inside out approach for cholesteatoma surgery. Int J Otorhinolaryngol Head Neck Surg 2018;4(2):481-9. 
[4] Roth TN, Haeusler R. Inside-out technique cholesteatoma surgery: a retrospective long-term analysis of 604 operated ears between 1992 and 2006. Otol Neurotol 2009;30(1):59-63.

[5] Maiti AB, Saha M, Patra S, et al. Canal wall down mastoidectomy-doctor dependent for whole life? Journal of Evolution of Medical and Dental Sciences 2016;5(63):4415.

[6] Kishore HK, Mallikarjuna Reddy C. Microbial study of pre and postoperative mastoid cavities: a comparative study. Int J Otorhinolaryngol Head Neck Surg 2017;3(1):37-41.

[7] Palva T, Hallstrom 0. Bacteriology of chronic otitis media. Arch Otolaryng 1965;82(4):35-64.

[8] Apte BC. Towards a dry ear. J Otolayngol 1960;12:917.

[9] Mishra SC, Kushwaha JLM, Yrat S. Microbial studies of postoperative mastoid cavities. Inidal J Otolaryngol 1990;42(2):63-5.

[10] Brook I, Finegold SM. Bacteriology of chronic otitis media. J Am Med Assoc 1979;241(5):487-8.

[11] Shim HJ, Park CH, Kim MG, et al. A pre- and postoperative bacteriological study of chronic suppurative otitis media. Infection 2010;38(6):447-52.

[12] An-ting X, Ming X, Han-Bing Z, et al. Bacteriolgy before and after total middle ear reconstruction. J Otol 2007;2(2):114-8.
[13] Elango S, Than T. Mastoiditis in Kelantan. Med J Malaysia 1995;50(3):233-6.

[14] Brook I. Management of chronic suppurative otitis media: superiority of therapy effective against anaerobic bacteria. Pediatr Infect Dis J 1994;13(3):188-93.

[15] Park DC, Lee SK, Cha CI, et al. Antimicrobial resistance of Staphylococcus from otorrhea in chronic suppurative otitis media and comparison with results of all isolated Staphylococci. Eur J Clin Microbiol Infect Dis 2008;27(7):571-7.

[16] Mittal R, Lisi CV, Gerring R, et al. Current concepts in the pathogenesis and treatment of chronic suppurative otitis media J Med Microbiol 2015;64(10):1103-16.

[17] Indudharan R, Haq JA, Aiyar S. Antibiotics in chronic suppurative otitis media: a bacteriologic study. Ann Otol Rhinol Laryngol 1999;108(5):440-5.

[18] Jang CH, Park SY. Emergence of ciprofloxacin-resistant pseudomonas in chronic suppurative otitis media. Clin Otolaryngol Allied Sci 2004;29(4):321-3.

[19] Dohar JE. Old and new ototopical agents for the acute and chronic draining ear. Seminars in Otitis Media Management 1998:1-14.

[20] Roland PS, Meyers AD. Chronic suppurative otitis media treatment \& management. Medscape 2017. 